\title{
Comparison of Composite Quality from PP Plastic with Recycled HDPE Through The Screw Extruder Process
}

\author{
Z Zulnazri*, W Atmaja, S Maliki, A Muarif, F Noviansyah, C Ramadhan \\ Department of Material Engineering, Universitas Malikussaleh, Aceh, Indonesia \\ *Corresponding author E-mail: wawan.190190002@mhs.unimal.ac.id
}

Manuscript received 23 Feb 2021; revised 2 March. 2021; accepted 15 March 2021. Date of publication 2 April 2021

\begin{abstract}
This study examines the composite quality of PP and HDPE plastic waste materials using Microfiber Oil palm empty fruit bunches $(O P E F B)$ as filler, the fiber used is $90 \mu \mathrm{m}$. The ratio of matrix: filler used is 60:40 and 70:30 for each type of PP and HDPE polymer. The method used is a melt blending screw extruder, where plastic and fiber materials are dissolved with a compatibilizer and then melt blended in an extruder by providing temperatures of 160 and $170{ }^{\circ} \mathrm{C}$. Tensile tests showed the strength of the PP composite with a filler ratio of 60:40 and 70:30, respectively, of $313.25 \mathrm{~N}$ and $336.35 \mathrm{~N}$, while the HDPE composite with a filler ratio of 60:40 and 70:30, respectively are $392.93 \mathrm{~N}$ and $187.90 \mathrm{~N}$. The maximum force required to break HDPE composites reaches 21.10 Mpa while for PP composites it reaches 18.56 Mpa. From the morphology of the PP and HDPE composite samples, the overall surface structure of HDPE looks regular with a width from 1 to $13.5 \mathrm{~mm}$. The PP composite shows a uniform and regularly arranged surface structure and the bond between the fibers and the filler looks more compatible but the surface pores are rougher. Heat resistance can be seen from the melting point of PP composites which can reach $163.81^{\circ} \mathrm{C}$ while HDPE composites only reach $134.21^{\circ} \mathrm{C}$.
\end{abstract}

Keywords: Plastic, Filler, OPEFB, Composite, PP, HDPE.

\section{Introduction}

One of the wastes that are often encountered around us is plastic waste. There are many negative effects of plastic waste, therefore an effective response is needed to overcome the problem of plastic waste. Efforts to reduce environmental pollution can be done by recycling plastics by depolymerizing certain types of plastic into other products, such as polyethylene terephthalate (PET) into terephthalic acid and ethylene glycol to make fibers, molding materials, and plastic cans. The type of high-density polyethylene (HDPE), which is usually used for milk packaging, is made into plastic bottles, toys, pipes, and other products. In general, there are four requirements so that a plastic waste can be processed by an industry, among others, waste must be homogeneous according to needs (seeds, pellets, powders, fractions), waste must be homogeneous, not polluted, and endeavored not to be oxidized[1].

On the other hand, the use of thermoplastic materials, such as polypropylene (PP) is very much consumed for packaging, such as film bags, bottles and containers for wrapping cheap materials in modern society. Thermoplastic composites made from lignocellulosic filler materials such as wood fiber and palm fruit bunches are currently being developed. Lignocellulose as a filler has many advantages over inorganic fillers, including low density, large deformability, flexibility, does not cause heat to the equipment during the ice process, low price, and comes from renewable resources [2].

Oil palm empty fruit bunches (OPEFB), have not been used as an economically valuable material, and cause problems for the environment because their decomposition takes time. OPEFB fiber is a lignocellulosic fiber that can be used as a filler to produce good polymer composites. Cellulose fiber processing has an effect on increasing the mechanical properties (tensile strength, modulus of elasticity, hardness) of the composite polymer. Treatment of cellulose fibers with agents enhances chemical bonds (especially amino groups) to improve the mechanical and chemical properties of water-immersed composite polymers[3] [4].

This study continues the research of Zulnazri, et al, 2020, who used HDPE polymer as a matrix with Oil Palm Empty Fruit Bunches as filler. Therefore, this study used PP polymer as a matrix compared to previous studies. In this study, a filler length of $90 \mu \mathrm{m}$ was used with matrix variations: filler 60: 40 and 70: 30. The analyzes were mechanical properties, thermal properties and morphology [5].

\section{Literature Review}

Composite manufacturing can be done through a two-stage process. In the first stage, the plastic raw materials are modified first. Then, the fillers are mixed together in a kneader and formed into a composite. Generally, this two-stage process results in a better product. The most influential operating conditions in the manufacture of composites are temperature, rotation speed, and stirring time. The more contact 
surface area of the mixed material or the smaller the particles, the better the mixing process and the higher the strength of the composite because the movement of particles in mixing is easier and more evenly distributed on the surface.

The solution technique used on a laboratory scale is done by dissolving polypropylene in xylene and adding fillers with various compositions and particle sizes. The mixture was refluxed with variations in time and added with and without dicumyl peroxide as an initiator and acrylic acid as a compatibilizer to determine the optimum compatibility. After the xylene is evaporated, the chemical composition of the mixture is analyzed and its physical properties are readily characterized. The variables tested were: temperature, residence time, die diameter, initiator concentration, compatibilizer agent content, and filler content. After the reaction process, the polymer mixture in the form of lumps or pellets was then put into a compression mold at a temperature of $180^{\circ} \mathrm{C}$ for 3 minutes without pressure, and an additional 3 minutes with a pressure of $100 \mathrm{kN}$ to form a polymer film., for microscopic, mechanical, and homogeneity testing [2].

Perform chemical modification of OPEFB with maleic anhydrous (MAH) dissolved in dimethylformamide at $90^{\circ} \mathrm{C}$. The MAH mixture and the filler mixture are processed in a Haake twin screw extruder (reverse). $2 \%$ dicumyl peroxide was added during the mixing process, operating temperature $165-180^{\circ} \mathrm{C}$ starting from the feed zone and end zone, with a screw speed of $35 \mathrm{rpm}$. The mixture is then extruded and made into pellets. The pellets were molded with dimensions of $17.0 \times 17.0 \times 0.3 \mathrm{~cm}$, then heated for 10 minutes at $180^{\circ} \mathrm{C}$ followed by hot pressing for 10 minutes. Cooling was carried out for 5 minutes before testing the flexural and impact properties [6].

Processed cellulose and EFB fibers were mixed in various ratios above 50\% with PP matrix which had been treated using a brabender twinscrew compounder at $180^{\circ} \mathrm{C}$ for 20 minutes, with a roller speed of $50 \mathrm{rpm}$. The printed composite sheet with a thickness of $1.2 .3 \mathrm{~mm}$ was produced at a temperature of $190{ }^{\circ} \mathrm{C}$ and a pressure of $150 \mathrm{~kg} / \mathrm{m} 2$. This process occurs preheating for 5 minutes 3 minutes, complete pressing in a hot press followed by cooling for 3 minutes under pressure from equipment equipped with a chiller. Then the mechanical and morphological tests of the composite were carried out [7]. The tensile strength of PP composites increased with the addition of OPEFB fiber above $20 \%$ and acrylic acid 3\% and dicumyl peroxide ratio of $0.01 \mathrm{~mol}$. Meanwhile, the elongation nature shows a downward trend. This observation also occurs when using a single screw extruder [7].

To improve the quality of the composite, a coupling agent was added to the thermoplastic polymer which aims to increase the interfacial adhesion between the thermoplastic and the fiber. The use of coupling agents will form cross-links between polymers and fibers so that the composite is stronger. The coupling agent that easily forms cross-links between polymers is maleic anhydrous (MAH). MAH is a compound that will form cross-links between polymers and other polymers. Cross-links can also occur between fiberglass and PP and HDPE. This cross-linking can occur properly if peroxide is added as an initiator which helps open the $\mathrm{CH}$ bonds in PP to form $+\mathrm{R}-\mathrm{OH}$ radicals [5].

\section{Methods}

HDPE and PP plastics as the matrix were pulverized by crusher, OPEFB fiber as filler was pulverized with a grinder mill with a size of 90 $\mu \mathrm{m}$. Modification of the manufacture of composites by mixing the matrix with fiber, $70 \mathrm{~g}$ of matrix moistened with $20 \mathrm{ml}$ of $0.25 \% \mathrm{BPO}$, stirred until homogeneous over the entire surface of the matrix, $30 \mathrm{~g}$ of filler was moistened with $20 \mathrm{ml}$ of $2.5 \%$ MAH. Furthermore, the matrix and filler are mixed and stirred until homogeneous, then the mold is put in a compression mold to obtain a composite specimen of ASTM D-638 type IV [8]. Composite quality tests carried out include Tensile Strength, SEM, and DSC.

\section{Results and Discussion}

\subsection{Tensile Strength}

Table 1 shows the tensile test results for HDPE and PP composites with matrix composition: 60:40 and 70:30 fibers, with a fiber size of $90 \mu \mathrm{m}$.

Table 1. The results of the HDPE and PP composite tensile strength tests

\begin{tabular}{ccccc}
\hline \multicolumn{1}{c}{ Name } & Max Force & Max Stress & Break Force & Break Disp. \\
\hline Parameter & $\begin{array}{c}\text { Calc. at Entire Area } \\
(\mathrm{N})\end{array}$ & $\begin{array}{c}\text { Calc. at Entire Area } \\
(\mathrm{MPa})\end{array}$ & $\begin{array}{c}\text { Sensitivity: } 10 \\
(\mathrm{~N})\end{array}$ & $\begin{array}{c}\text { Sensitivity: } 10 \\
(\mathrm{~mm})\end{array}$ \\
\hline HDPE 60:40 & 392.93 & 21.11 & 391.56 & 1.87 \\
HDPE 70:30 & 187.90 & 9.58 & 95.67 & 1.49 \\
PP 60:40 & 313.25 & 14.03 & 155.09 & 1.42 \\
PP 70:30 & 336.35 & 18.56 & 322.79 & 1.24 \\
\hline
\end{tabular}

Figure 1 in graph (a) shows the graph of the results of the HDPE composite tensile test with a matrix/filler ratio of 60: 40 and a filler size of $90 \mu \mathrm{m}$. Table 1 and Figure 1 (a) explain that the maximum tensile strength in this treatment is $21.11 \mathrm{MPa}$ and the composite will fracture at a force of $391.56 \mathrm{~N}$. Figure 1 (b) shows the graph of the tensile test results for HDPE composites with a matrix/filler ratio of $70: 30$ and the filler size is $90 \mu \mathrm{m}$. Table 1 and Figure 1 (b) explain that the maximum tensile strength in this treatment is $9.58 \mathrm{MPa}$ and the composite will fracture at a force of $95.67 \mathrm{~N}$. 


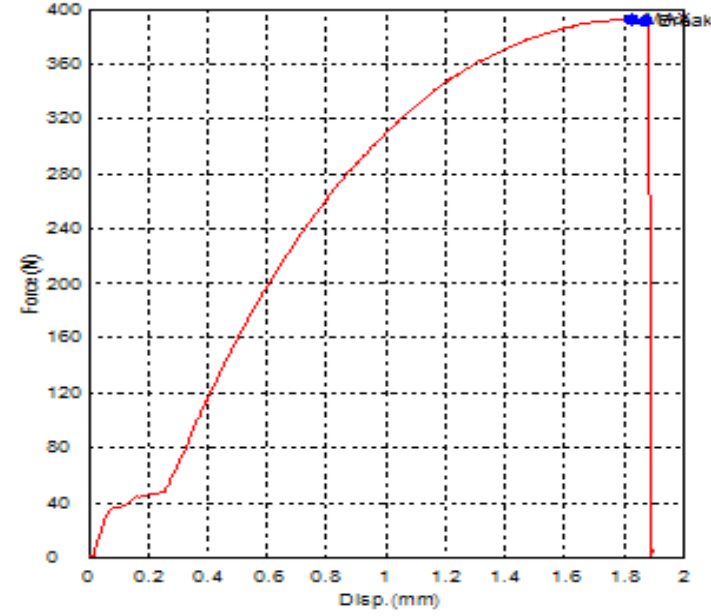

(a)

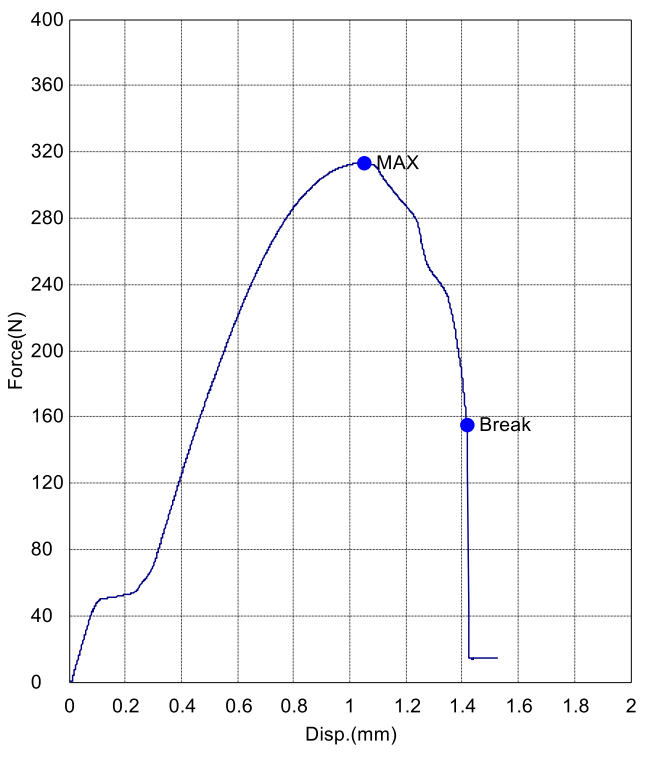

(c)

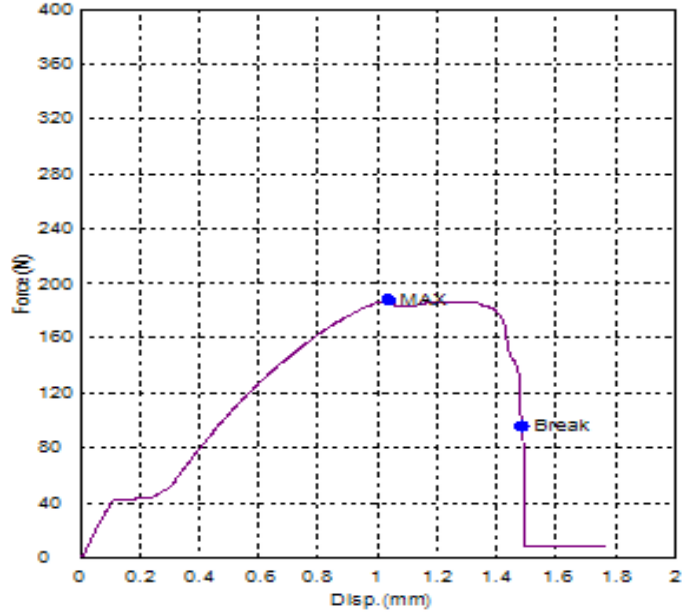

(b)

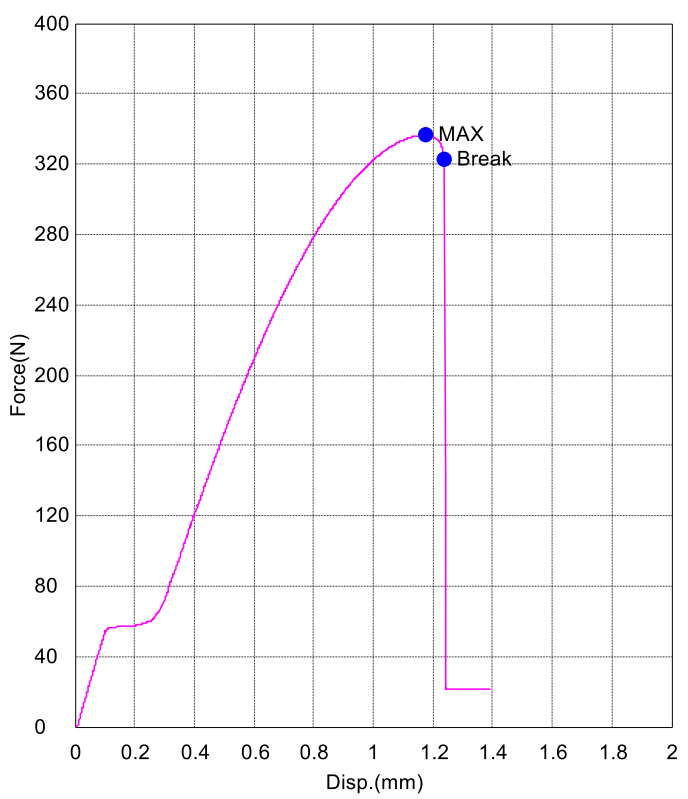

(d)

Fig 1. Composite tensile test graph, (a) HDPE : filler (60 : 40), (b) HDPE : filler (70 : 30), (c) PP : filler (60:40), (d) PP : filler (70:30)

Based on the above analysis, it can be seen that the maximum tensile strength of HDPE composites with a matrix: filler ratio of 60: 40 is 21.11 MPa and the composite will fracture at a force of $391.56 \mathrm{~N}$. Analysis of PP composites shows that the maximum tensile strength owned by PP composites with a matrix: filler ratio treatment of 70: 30 which is $18.56 \mathrm{MPa}$ and the composite will fracture at a force of 322.79 N. It is clearly seen in Figure 1 that HDPE composites are more flexible than PP composites where if the composite is If given a load, the composite will experience flexibility before fracture. This indicates that the bond strength between the various components in the composite has a significant effect on its properties. The bond between the HDPE matrix is stronger than the bond between the PP matrix and the OPEFB filler.

\subsection{Scanning Electron Microscopy (SEM)}

The morphology and dimensions of the two HDPE and PP materials after mixing with OPEFB filler were determined by SEM analysis as shown in the following figure.

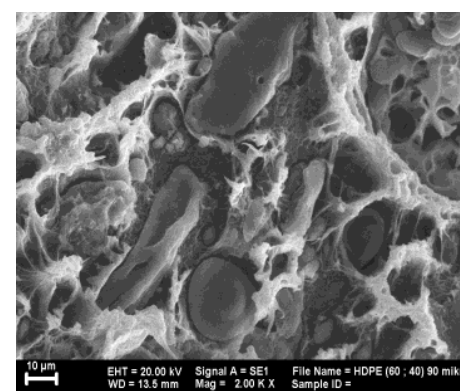

(a)

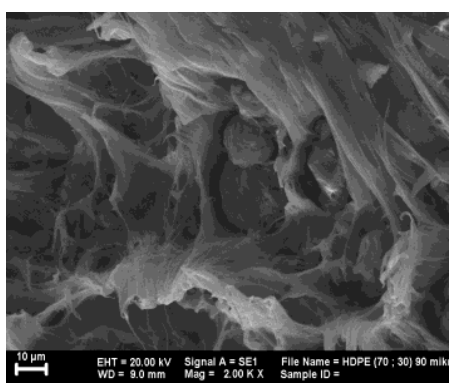

(b) 


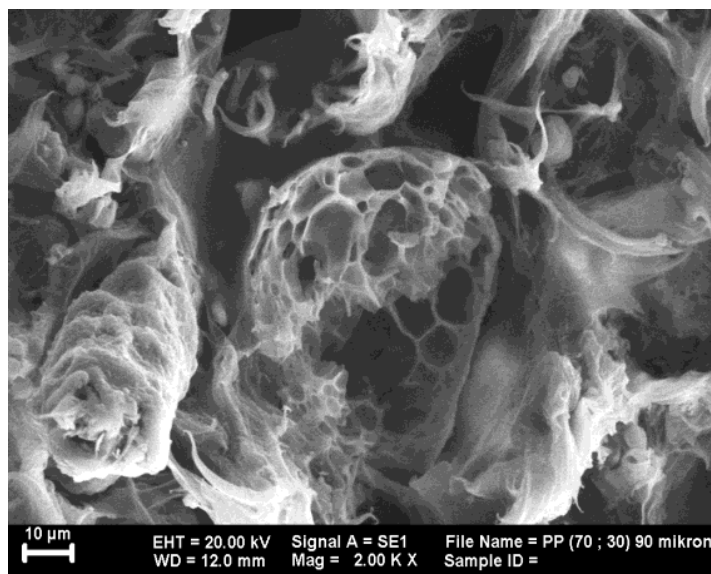

(c)

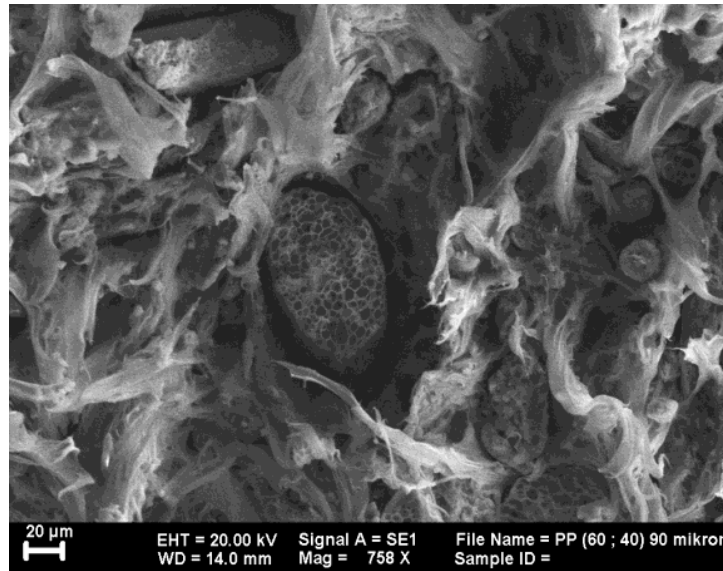

(d)

Fig 2. Morphological analysis by SEM, (a) HDPE composite (60:40), (b) HDPE composite (70:30), (c) PP composite (60:40), (d) PP composite (70:30) $90 \mu \mathrm{m}$

The overall surface structure of HDPE looks regular with a width from 1 to $13.5 \mathrm{~mm}$. Scanning electron micrographs show the morphological changes of the composites due to the size and fineness of the fibers. So that the size of the fineness of the fiber as a filler needs to be considered to provide a better surface structure, as shown in Figures 2 (a) and (b) show that the surface structure tends to be less uniform. The fibers shown in this figure show the typical shape of the lignocellulosic morphology of OPEFB.

Same as in HDPE composites, the results of SEM analysis on PP composites showed changes in the morphology of the composite because it was influenced by the size and fineness of the fiber. However, PP composites always provide a surface morphology that is not neatly arranged with fibers due to the elastic and very tough properties of PP so that it can cover the surface of the fiber. The size of the fineness of the fiber as a filler needs to be considered to provide a better surface structure, as shown in Figures 2 (c) and (d) showing a uniform and regularly arranged surface structure and the bond between the fiber and the filler looks more compatible but the surface pores more rough.

\subsection{Differential Scanning Calorymeter (DSC)}

The DSC (Differential Scanning Calorymeter) test is carried out to see the melting point of the composite to heat and to see the amount of heat needed to reach the melting point. Composite materials experience a decrease in melting point along with the addition of filler, this occurs because the polymer chain is physically degraded, meaning that the polymer chain is pushed by the filler material, thereby damaging the polymer molecular chain bonds.

The melting point and decomposition temperature of HDPE and PP composites is not a single price but lies in a certain range. Figures 3 (a) and (b) show that the first peak provides information about the melting point of the material, while the second peak provides information about the decomposition temperature and the general character of the curve. The melting point is influenced by the shape of the molecular symmetry and the molecular weight of the polymer compound and the degree of crystallinity of the composite material, the higher the degree of crystallinity of the composite material, the higher the melting point of the composite material, and vice versa.

In Figure 3 (a) above, it is clear that the melting point of the tested composites, where the melting point of HDPE-OPEFB composites can reach $134.21^{\circ} \mathrm{C}$. The decomposition temperature is the temperature at which the material begins to change in composition and there is a shift in the molecules contained in the constituent materials. From Figure 3 (b) it can be seen that the PP-filler composite has a point of $163.81^{\circ} \mathrm{C}$.

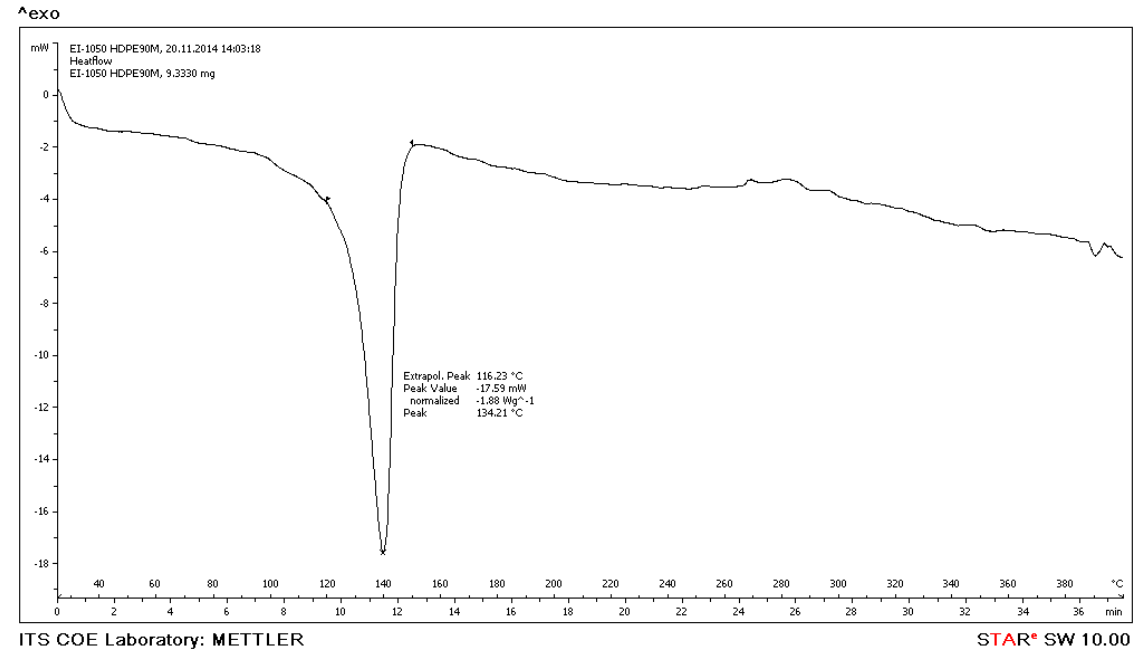




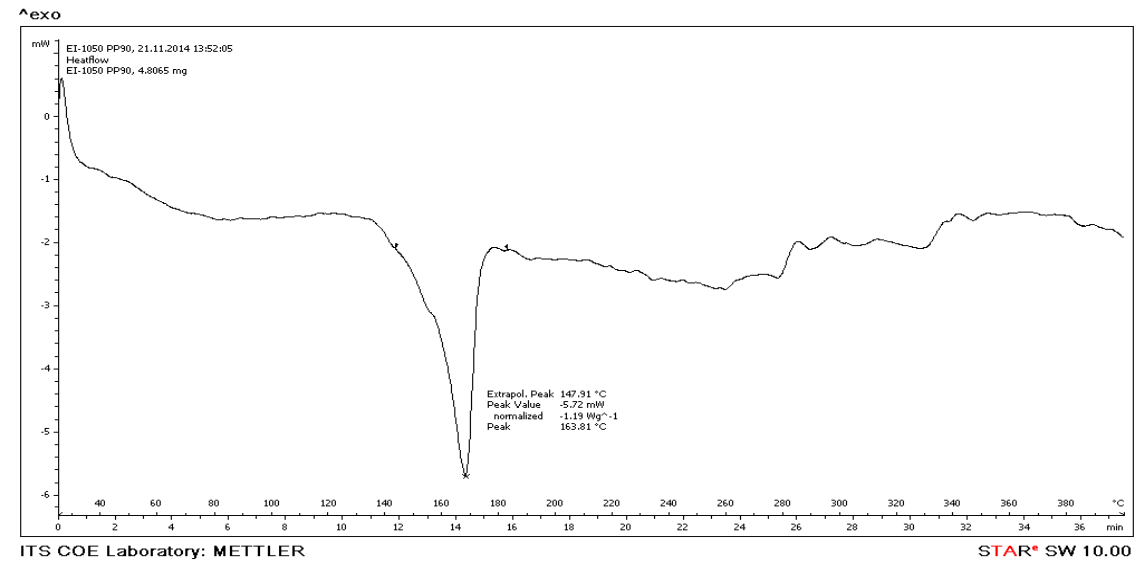

Fig 3. Thermal test analysis with DSC (a) HDPE composite (70:30), (b) PP composite (70:30)

\section{Conclusion}

From the research that has been done, HDPE composites have better quality than PP composites when viewed from the tensile test and surface morphology. This is indicated by the maximum force reaching $21.10 \mathrm{MPa}$ for HDPE while for PP composite the maximum force reaching 18.56 MPa. For SEM results, HDPE's surface structure is neater than PP. And the heat resistance of HDPE and PP composites is also different, for PP composites have greater heat resistance than HDPE composites.

\section{References}

[1] Z. Zulnazri, R. Dewi, and H. F. Sangian, "The Effect of Fibre Composition on the Strenght of Microcomposite from Recycled Polypropilene Plastics,” Int. J. Psychosoc. Rehabil. Conf. Spec. Issue, vol. 24, no. Special Issue 2, pp. 403-413, 2020.

[2] B. Wirjosentono, P. Guritno, and H. Ismail, "Oil Palm Empty Fruit Bunch Filled Polypropylene Composites," Int. J. Polym. Mater., vol. 53, no. 4, pp. 295-306, 2004, doi: 10.1080/00914030490429942.

[3] Z. Zulnazri and R. Dewi, "Microcomposites from Recycled Polypropylene Plastic with Reinforcement Fiber of Oil Palm Empty Fruit Bunches," Int. Conf. Multidiscip. Eng. Adv. Eng. Hum. Prosper. Environ. Sustain., vol. 1, pp. 81-85, 2018.

[4] P. V. Notingher, D. Panaitescu, Z. Vuluga, M. Iorga, H. Paven, and D. Florea, "The Effect of Water on Electrical Properties of Polymer Composites with Cellulose Fibers," J. Optoelectron. Adv. Mater., vol. 8, no. 2, pp. 687-689, 2006.

[5] Z. Zulnazri, R. Dewi, and N. Sylvia, "Modification of Recycled HDPE Composite with OPEFB Microfibers Through The Melt Blend Extruder Process," J. IPTEK, vol. 24, pp. 105-112, 2020, doi: 10.31284/j.iptek.2020.v24i2.92.

[6] H. D. Rozman, M. J. Saad, and Z. A. M. Ishak, "Flexural and Impact Properties of Oil Palm Empty Fruit Bunch (EFB)-Polypropylene Composites-The Effect of Maleic Anhydride Chemical Modification of EFB," Polym. Test., vol. 22, pp. 335-341, 2003, doi: $10.1016 / \mathrm{S}$.

[7] M. Khalid, C. T. Ratnam, T. G. Chuah, S. Ali, and T. S. Y. Choong, "Comparative Study of Polypropylene Composites Reinforced with Oil Palm Empty Fruit Bunch Fiber and Oil Palm derived Cellulose,” Mater. Des., vol. 29, pp. 173-178, 2008, doi: 10.1016/j.matdes.2006.11.002.

[8] D.-14 ASTM, "Standard Test Method for Tensile Properties of Plastics," ASTM Int. West Conshohocken, vol. 8, pp. 1-17, 2014, doi: 10.1520/D0638-14. 